\title{
Determination of Thermal Stresses in A Thin Clamped Hollow Disk under Unsteady Temperature Field due To Point Heat Source
}

\author{
G.D. $\operatorname{Kedar}^{1}$, K.C. Deshmukh ${ }^{2}$ \\ ${ }^{l}$ Department of mathematics, Kamla Nehru Mahavidyalaya, Sakkardara, Nagpur, Maharashtra, India. \\ ${ }^{2}$ Department of mathematics, Nagpur University, Nagpur, Maharashtra, India
}

\begin{abstract}
This paper deals with the determination of thermal stresses in a thin clamped hollow disk under unsteady temperature field due to point heat source situated at centre along radial and axial direction within it. A thin hollow disk is considered having arbitrary initial temperature and is subjected to arbitrary heat flux at the outer circular boundary; whereas inner boundary is at zero heat flux. Also, the upper and lower surfaces of the disk are at zero temperature. The inner and outer edges of the disk are clamped. The governing heat conduction equation has been solved by the method of integral transform technique. The results are obtained in a series form in terms of Bessel's functions. The results have been computed numerically and illustrated graphically.
\end{abstract}

Keywords:Heat Conduction, Point Heat Source, Thermal Stresses, clamped hollow disk, Unsteady Temperature.

\section{Introduction}

During the last century the theory of elasticity has found of considerable applications in the solution of engineering problems. There are many cases in which the elementary methods of strength of materials are inadequate to furnish satisfactory information regarding stress distribution in engineering structures and recourse must be made to the more powerful methods of the theory of elasticity. The elementary theory is insufficient to give information regarding local stresses near the loads and the supports of beams. It fails also in the cases when the stress distribution in bodies, all the dimensions of which are of the same order, has to be investigated. The stresses in rollers and in balls of bearing can be found only by using the theory of elasticity. During recent years consideration progress has been made in solving such practically important problems. In cases where a rigorous solution cannot be readily obtained, approximate methods have been developed. In some cases solutions have been obtained by using experimental methods. These important quantities were conventionally obtained by experimental methods which normally involve delicate and sophisticated equipments. The method of direct determination of stresses and the use of the compatibility equations in terms of stress components have been applied for solving various problems and in many cases the energy method of solution of elasticity problems has been used. Roy Choudhuri [1], determined the quasi-static thermal stresses in thin circular disk subjected to transient temperature along the circumference of a circle over the upper face with lower face at zero temperature and fixed circular edge thermally insulated. Gogulwar and Deshmukh [2] thermal stresses in a thin circular plate with heat sources.

Recently Kulkarni et al. [3], dealt with the determination of displacement and thermal stresses in a thin hollow circular disk defined by $a \leq r \leq b$ due to instantaneous line heat source situated at the centre of the circular disk along the radial direction and most recently, Deshmukh et al. [4], studied non-homogeneous steady state heat conduction problem in a thin circular plate and discussed its thermal stresses due to its internal heat generation at a constant rate.

This paper deals with the determination of thermal stresses in a thin clamped hollow disk under unsteady temperature field due to point heat source situated at centre along radial and axial direction within it. A thin hollow disk is considered having arbitrary initial temperature and is subjected to arbitrary heat flux at the outer circular boundary; whereas inner boundary is at zero heat flux. Also, the upper surface $z=h$ and the lower surface $z=0$ of the disk are at zero temperature. The inner and outer edges of the disk are clamped. The governing heat conduction equation has been solved by the method of integral transform technique. The results are obtained in a series form in terms of Bessel's functions. The results have been computed numerically and illustrated graphically.

\section{Formulation Of The Problem}

Consider a thin hollow disk of thickness $h$ occupying space $D: a \leq r \leq b, 0 \leq z \leq h$ as shown in Fig 1. Initially, the disk is kept at arbitrary temperature $F(r, z)$. The inner circular boundary $r=a$ is at zero 
temperature whereas the heat flux $\frac{Q(z, t)}{k}$ is applied on the outer circular boundary $r=b$. Also the upper surface $z=h$ and the lower surface $z=0$ of the disk are at zero temperature. For time $t>0$, heat is generated within the thin hollow disk at the rate $g(r, z, t)$. Under these conditions, the thermal stresses in a thin hollow disk due to heat generation are required to be determined.

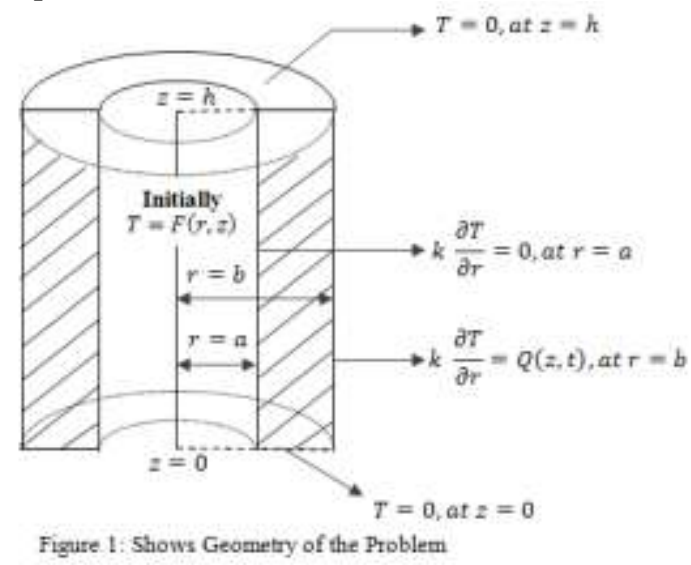

Following Deshmukh et al [1], the differential equation governing the displacement function $\psi(r, z, t)$ is

$$
\frac{\partial^{2} \psi}{\partial r^{2}}+\frac{1}{r} \frac{\partial \psi}{\partial r}=(1+v) a_{t} T
$$

with,

$$
\frac{\partial \psi}{\partial r}=0 \quad \text { at } r=a, r=b, \text { for } t \geq 0,0 \leq z \leq h .
$$

where, $v$ is Poisson's ratio and $a_{t}$ is the linear coefficient of thermal expansion of the material. $T=T(r, z, t)$ is the temperature of the thin hollow disk at time $t$ satisfying the equation

$$
\frac{\partial^{2} T}{\partial r^{2}}+\frac{1}{r} \frac{\partial T}{\partial r}+\frac{\partial^{2} T}{\partial z^{2}}+\frac{g(r, z, t)}{K}=\frac{1}{\alpha} \frac{\partial T}{\partial t}
$$

with the boundary conditions

$$
\begin{array}{cccrl}
\frac{\partial T}{\partial r}=0, & \text { at } & & r=a, t>0,0 \leq z \leq h \\
k \frac{\partial T}{\partial r}=Q(z, t), & \text { at } & & r=b, t>0,0 \leq z \leq h \\
T(r, z, t)=0, & \text { at } & & z=0, t>0, a \leq r \leq b . \\
T(r, z, t)=0, & \text { at } & & z=h, t>0, a \leq r \leq b \\
\text { Initially } & & & \\
T(r, z, t)=F(r, z), & \text { at } & & t=0, a \leq r \leq b, 0 \leq z<\infty
\end{array}
$$

where, $\alpha, K$ and $k$ are the thermal diffusivity, thermal conductivity and heat transfer coefficient of the material of the hollow disk respectively. The stress function $\sigma_{r r}$ and $\sigma_{\theta \theta}$ are given by

$$
\begin{aligned}
& \sigma_{r r}=-2 \mu \frac{1}{r} \frac{\partial \psi}{\partial r} \\
& \sigma_{\theta \theta}=-2 \mu \frac{\partial^{2} \psi}{\partial r^{2}}
\end{aligned}
$$

where, $\mu$ is the Lamé constant.

For traction free surfaces,

$$
\sigma_{r r}=0, \quad \text { at } r=a \text { and } r=b
$$

Also, in the planer state of stress within thin disk

$$
\sigma_{r z}=\sigma_{z z}=\sigma_{z \theta}=0 \text {. }
$$

Equations (1) to (12) constitute the mathematical formulation of the problem.

\subsection{Temperature Distribution Function}

\section{Solution}

The partial derivative with respect to the $z$ variable is removed by means of Fourier transform and the partial derivative with respect to the $r$ variable is removed by means of Hankel transform. Since the range of the $z$-variable is $0 \leq z \leq h$, one defines the Fourier transform and the inversion formula of the temperature function $T(r, z, t)$ with respect to the $z$-variable as suggested by Ozisik [5] 


$$
\begin{aligned}
& \bar{T}\left(r, \eta_{p}, t\right)=\int_{z=0}^{h} K\left(\eta_{p}, z\right) T(r, z, t) d z \\
& T(r, z, t)=\sum_{p=1}^{\infty} K\left(\eta_{p}, z\right) \bar{T}\left(r, \eta_{p}, t\right)
\end{aligned}
$$

where, $K(\eta, z)$ is the kernel which is obtained from the eigenvalue problem. Using variable separation method and then solving eigen value problem one obtains the kernel $K(\eta, z)$. Applying Fourier sine transform (13) to the system (3)-(8), reduces to a system which includes a differential operator with respect to the $r$ variable. The Hankel transform is suitable to remove the $r$ variable in the reduced system. One defines the Hankel transform and inversion formula of the function $\bar{T}(r, \eta, t)$ in the range $a \leq r \leq b$ as

$$
\begin{aligned}
& \overline{\bar{T}}\left(\lambda_{m}, \eta_{p}, t\right)=\int_{r=a}^{b} r K_{0}\left(\lambda_{m}, r\right) \bar{T}\left(r, \eta_{p}, t\right) d r \\
& \bar{T}\left(r, \eta_{p}, t\right)=\sum_{m=1}^{\infty} K_{0}\left(\lambda_{m}, r\right) \overline{\bar{T}}\left(\lambda_{m}, \eta_{p}, t\right)
\end{aligned}
$$

Now on applying Hankel transform defined as in (15) to the reduced system, the partial differential equation of heat conduction is reduced to an ordinary linear differential equation for double transform of the temperature $T(r, z, t)$. Solving the ordinary differential equation and the resulting double transform of temperature is inverted by means of the inversion formulas (16) and (14); one finds the solution of the present boundary value problem of heat conduction. The temperature distribution obtained as

$$
\begin{aligned}
& T(r, z, t)=\sum_{p=1}^{\infty} \sum_{m=1}^{\infty}\left\{K_{o}\left(\lambda_{m}, r\right) K\left(\eta_{p}, z\right) e^{-\alpha\left(\lambda_{m}^{2}+\eta^{2}\right) t}\right\}\left\{\int_{a}^{b} \int_{0}^{h} r^{\prime} K_{o}\left(\lambda_{m}, r^{\prime}\right) K\left(\eta_{p}, z^{\prime}\right) F\left(r^{\prime}, z^{\prime}\right) d r^{\prime} d z^{\prime}\right. \\
& +\frac{\alpha}{K} \int_{t^{\prime}=0}^{t} e^{\alpha\left(\lambda_{m}^{2}+\eta^{2}\right) t^{\prime}} \llbracket \int_{a}^{b} \int_{0}^{h} r^{\prime} K_{o}\left(\lambda_{m}, r^{\prime}\right) K\left(\eta_{p}, z^{\prime}\right) g\left(r^{\prime}, z^{\prime}, t^{\prime}\right) d r^{\prime} d z^{\prime} \\
& \left.+b K_{o}\left(\lambda_{m}, b\right) \int_{0}^{h} K\left(\eta_{p}, z^{\prime}\right) Q\left(z^{\prime}, t^{\prime}\right) d z^{\prime} \rrbracket d t^{\prime}\right\}
\end{aligned}
$$

where, the kernels are defined as

$$
K\left(\eta_{p}, z\right)=\sqrt{\frac{2}{h}} \sin \eta_{p} z
$$

and

$$
K_{o}\left(\lambda_{m}, r\right)=\frac{\pi}{\sqrt{2}} \frac{\lambda_{m} Y_{1}\left(\lambda_{m} b\right) J_{1}\left(\lambda_{m} b\right)}{\sqrt{1-\frac{J_{1}^{2}\left(\lambda_{m} b\right)}{J_{1}^{2}\left(\lambda_{m} a\right)}}}\left[\frac{J_{0}\left(\lambda_{m} r\right)}{J_{1}\left(\lambda_{m} b\right)}-\frac{Y_{0}\left(\lambda_{m} r\right)}{Y_{1}\left(\lambda_{m} b\right)}\right]
$$

where, $\lambda_{m}^{\prime s}$ are the positive roots of the transcendental equation

$$
\frac{J_{1}\left(\lambda_{m} a\right)}{J_{1}\left(\lambda_{m} b\right)}-\frac{Y_{1}\left(\lambda_{m} a\right)}{Y_{1}\left(\lambda_{m} b\right)}=0
$$

\subsection{Displacement Function and Thermal Stresses}

Substituting the temperature distribution obtained in equation (17) in the equation (1) one obtained displacement function as

$$
\begin{aligned}
\frac{\psi}{(1+v) a_{t}}=\sum_{p=1}^{\infty} \sum_{m=1}^{\infty} & \llbracket \frac{K_{o}\left(\lambda_{m}, b\right)-K_{o}\left(\lambda_{m}, r\right)}{\lambda_{m}^{2}} \rrbracket K\left(\eta_{p}, z\right) e^{-\alpha\left(\lambda_{m}^{2}+\eta^{2}\right) t} \\
& \times\left\{\int_{a}^{b} \int_{0}^{h} r^{\prime} K_{o}\left(\lambda_{m}, r\right) K\left(\eta_{p}, z^{\prime}\right) F\left(r^{\prime}, z^{\prime}\right) d r^{\prime} d z^{\prime}\right. \\
& +\frac{\alpha}{K} \int_{t^{\prime}=0}^{t} e^{\alpha\left(\lambda_{m}^{2}+\eta^{2}\right) t^{\prime}} \llbracket \int_{a}^{b} \int_{0}^{h} r^{\prime} K_{o}\left(\lambda_{m}, r^{\prime}\right) K\left(\eta_{p}, z^{\prime}\right) g\left(r^{\prime}, z^{\prime}, t^{\prime}\right) d r^{\prime} d z^{\prime}
\end{aligned}
$$




$$
\left.+b K_{o}\left(\lambda_{m}, b\right) \int_{0}^{h} K\left(\eta_{p}, z^{\prime}\right) Q\left(z^{\prime}, t^{\prime}\right) d z^{\prime} \rrbracket d t^{\prime}\right\}
$$

Using this expression in equations (9) and (10), one obtains radial stress function and angular stress function as

$$
\begin{aligned}
& \sigma_{r r}=-2 \mu(1+v) a_{t} \sum_{p=1}^{\infty} \sum_{m=1}^{\infty} \llbracket \frac{K_{1}\left(\lambda_{m}, r\right)}{\lambda_{m} r} \rrbracket K\left(\eta_{p}, z\right) e^{-\alpha\left(\lambda_{m}^{2}+\eta^{2}\right) t} \\
& \times\left\{\int_{a}^{b} \int_{0}^{h} r^{\prime} K_{o}\left(\lambda_{m}, r^{\prime}\right) K\left(\eta_{p}, z^{\prime}\right) F\left(r^{\prime}, z^{\prime}\right) d r^{\prime} d z^{\prime}\right. \\
& +\frac{\alpha}{K} \int_{t^{\prime}=0}^{t} e^{\alpha\left(\lambda_{m}^{2}+\eta^{2}\right) t^{\prime}} \| \int_{a}^{b} \int_{0}^{h} r^{\prime} K_{o}\left(\lambda_{m}, r^{\prime}\right) K\left(\eta_{p}, z^{\prime}\right) g\left(r^{\prime}, z^{\prime}, t^{\prime}\right) d r^{\prime} d z^{\prime} \\
& \left.+b K_{o}\left(\lambda_{m}, b\right) \int_{0}^{h} K\left(\eta_{p}, z^{\prime}\right) Q\left(z^{\prime}, t^{\prime}\right) d z^{\prime} \rrbracket d t^{\prime}\right\} \\
& \sigma_{\theta \theta}=-2 \mu(1+v) a_{t} \sum_{p=1}^{\infty} \sum_{m=1}^{\infty}\left[K\left(\eta_{p}, z\right) \llbracket K_{0}\left(\lambda_{m}, r\right)-\frac{K_{1}\left(\lambda_{m}, r\right)}{\lambda_{m} r} \rrbracket\right. \\
& \times e^{-\alpha\left(\lambda_{m}^{2}+\eta^{2}\right) t} \times\left\{\int_{a}^{b} \int_{0}^{h} r^{\prime} K_{o}\left(\lambda_{m}, r^{\prime}\right) K\left(\eta_{p}, z^{\prime}\right) F\left(r^{\prime}, z^{\prime}\right) d r^{\prime} d z^{\prime}\right. \\
& +\frac{\alpha}{K} \int_{t^{\prime}=0}^{t} e^{\alpha\left(\lambda_{m}^{2}+\eta^{2}\right) t^{\prime}} \llbracket \int_{a}^{b} \int_{0}^{h} r^{\prime} K_{o}\left(\lambda_{m}, r^{\prime}\right) K\left(\eta_{p}, z^{\prime}\right) g\left(r^{\prime}, z^{\prime}, t^{\prime}\right) d r^{\prime} d z^{\prime} \\
& \left.+b K_{o}\left(\lambda_{m}, b\right) \int_{0}^{h} K\left(\eta_{p}, z^{\prime}\right) Q\left(z^{\prime}, t^{\prime}\right) d z^{\prime} \rrbracket d t^{\prime}\right\}
\end{aligned}
$$

\section{Special Case And Numerical Calculations}

To construct the mathematical thermoelastic behavior of the thin hollow disk, one considers the following parameters:

Set,

$F(r, z)=\left(r^{2}-a^{2}\right) \times r^{2} \times\left(z^{2}-h^{2}\right) \times z^{2}$

$Q(z, t)=\left(z^{2}-h^{2}\right)^{2} \times z^{2} \times e^{-\omega t}$

$g(r, z, t)=g_{p i} \times \delta\left(r-r_{1}\right) \times \delta\left(z-z_{1}\right) \times \delta(t-\tau)$

With, $\omega=10, t \rightarrow \tau=5 s$ and $g_{p i}=50$. Here, $r$ is the radius measured in meter and $\delta$ is the Dirac-delta function.

The heat source $g(r, z, t)$ is an instantaneous point heat source $g_{p i}=50 \mathrm{Btu} / \mathrm{hr} . \mathrm{m}$ situated at the centre of the disk along radial and axial direction and released its heat instantaneously at the time $t=\tau=5 \mathrm{~s}$.

A disk with its thickness less than $1 / 5$ of its smallest dimension is known as a thin disk. While if the thickness of disk exceed 1/5 of its smallest dimension it is termed as thick disk [6]. Here one considers a thin hollow disk, for which the dimensions are considered as follows.

\section{Dimensions of the hollow disk}

Outer radius of the disk $\quad b=2 \mathrm{~m}$

Inner radius of the disk $\quad a=1 \mathrm{~m}$

Thickness of cylinder disk $h=0.4 \mathrm{~m}$

Constants for special Case $r_{1}=1.5 \mathrm{~m}, z_{1}=0.2 \mathrm{~m}$

\section{Material properties}

The numerical calculation has been carried out for a Copper (pure) thin hollow disk with the material properties. Thermal conductivity $\quad k=386 \mathrm{~W} \mathrm{~m}^{-1} k^{-1}$

Thermal diffusivity

Density

$\alpha=112.34 \times 10^{-6} \mathrm{~m}^{2} \mathrm{~s}^{-1}$

Specific heat

$c_{p}=383 \mathrm{~J} \mathrm{~kg}^{-1} \mathrm{~K}^{-1}$

$$
\rho=8954 \mathrm{~kg} \mathrm{~m}^{-3}
$$


Coefficient of linear thermal expansion,

$$
a_{t}=16.5 \times 10^{-6} K^{-1}
$$

Poisson ratio

$$
v=0.35
$$

Lamé constant

$$
\mu=26.67
$$

\section{Roots of the transcendental equation}

The first five positive roots of the transcendental equation (16) are

$$
\lambda_{1}=3.1965, \quad \lambda_{2}=6.3123, \quad \lambda_{3}=9.4445, \quad \lambda_{4}=12.5812, \quad \lambda_{5}=15.7199
$$

Set for convenience,

$X=\frac{\pi}{\sqrt{h}}, \quad A=-(1+v) a_{t} \frac{\pi}{\sqrt{h}}, \quad B=\frac{2 \mu(1+v) a_{t} \pi}{\sqrt{h}}$

The numerical calculation has been carried out with the help of computational mathematical software Mathcad2000 professional and the graphs are plotted with the help of Excel (MS Office -2007).

From Fig 2, it can be observed that due to point heat source situated at centre along radial and axial direction the temperature distribution function decreases along radial direction in the region $1 \leq r \leq 1.5 \mathrm{~m}$ and then it increases towards outer curved surface of the disk. From Fig 3, it can be observed that the displacement potential function decreases along radial direction in the region $1 \leq r \leq 1.5 \mathrm{~m}$ and then it increases towards outer curved surface of the disk. It can be noticed that large displacement occurs at the inner boundary surface of the disk and it is proportional to the temperature distribution.

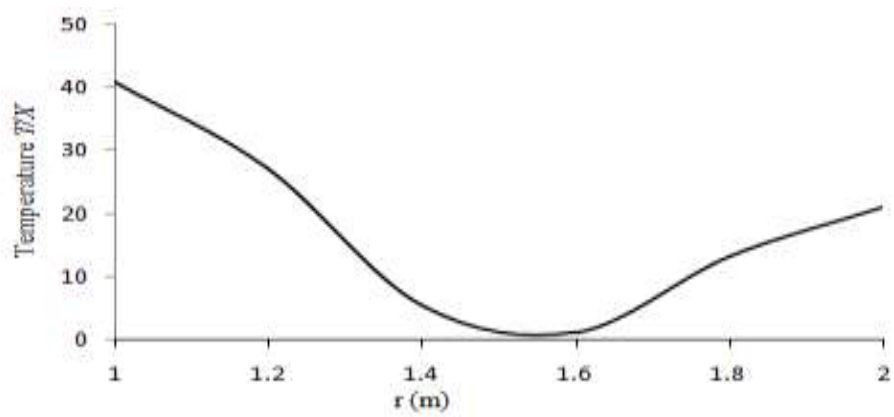

Figure 2: Temperature distribution along radial direction

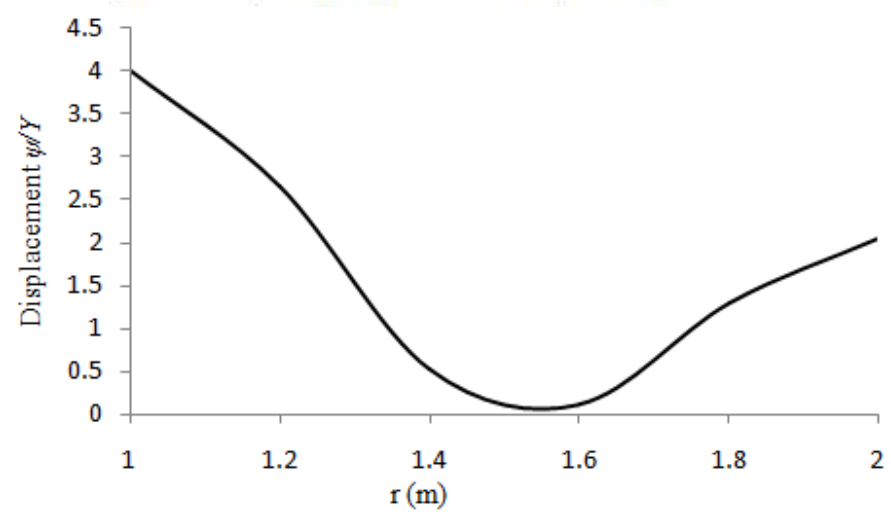

Figure 3: displacement potential function along radial direction

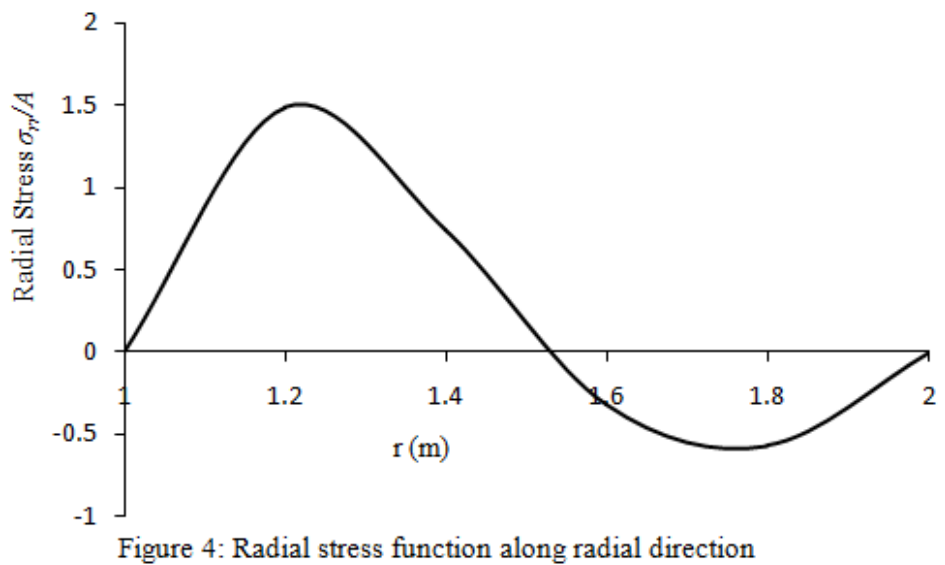




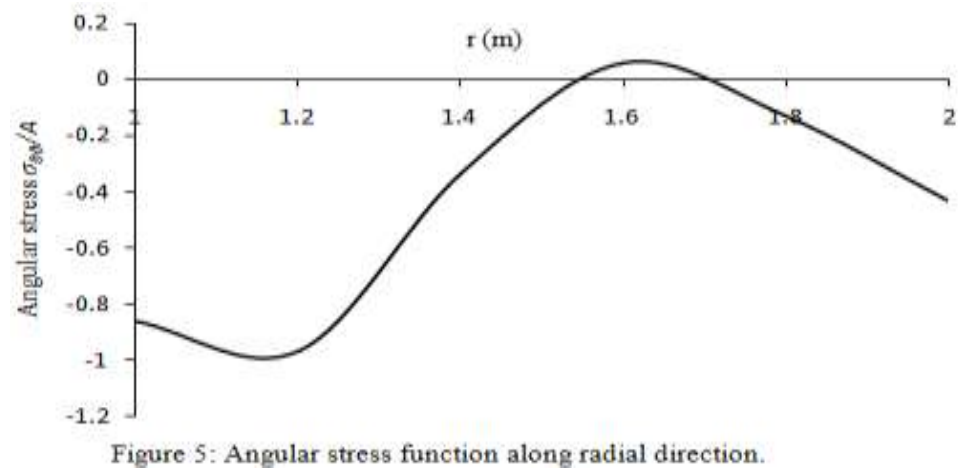

From Fig 4, it is observed that for traction free surface $r=2 \mathrm{~m}$ as well as at centre radial stress is zero. Radial stress increases in the central part $1 \leq r \leq 1.2 \mathrm{~m}$ and then decreases in the annular region $1.2 \leq r \leq 1.8 \mathrm{~m}$. In Fig 5 , it can be observed that the angular stress function increases from inner circular surface to outer circular face. Angular stress increases rapidly in the region $1.2 \leq r \leq 1.6 \mathrm{~m}$.

\section{Conclusion}

This paper deals with the determination of thermal stresses in a thin clamped hollow disk under unsteady temperature field due to internal heat generation within it. A thin hollow disk is considered having arbitrary initial temperature and subjected to heat flux at the outer boundary, where as inner circular boundary is at zero heat flux. Also, the upper surface $z=h$ and the lower surface $z=0$ of the disk are at zero temperature. As a special case mathematical model is constructed for Copper (pure) thin hollow disk with the material properties specified as above. The heat source is an instantaneous point heat source of strength $g_{p i}$ situated at $r=1.5, z=0.2$ and released its heat spontaneously at the time $t=\tau=5 \mathrm{~s}$.

One cans summaries that due to small thickness the stress components and displacement occurs near heated region. Due to heat generation within the thin hollow disk, the radial stress develops tensile stresses where as angular stress develops compressive stresses. Also it can be observed that heat flow and direction of body displacement are parallel and proportionate.

The results obtained here are useful in engineering problems particularly in the determination of state of stress in thin hollow disk. Also any particular case of special interest can be derived by assigning suitable values to the parameters and functions in the expressions (17), (21), (22) and (23).

\section{Acknowledgement}

The authors are thankful to University Grants Commission, New Delhi to provide the partial financial assistance under major research project scheme.

\section{References}

[1] Roy Choudhuri S.K., A note on the quasi-static stress in thin circular plate due to transient temperature applied along the circumference of a circle over the upper face, Bull. Acad. Polon. Sci., Ser. Sci. Techn., 20, 21, (1972).

[2] Gogulwar V.S. and Deshmukh K.C., Thermal stresses in a thin circular plate with heat sources, Journal of Indian Academy of Mathematics, 27 (1), 129-141, (2005).

[3] Kulkarni V. S., Deshmukh K. C. and Warbhe S. D., Quasi-Static thermal stresses due to heat generation in a thin hollow circular disk, J. Thermal Stresses, 31(8), 698-705, (2008).

[4] Deshmukh K.C., Warbhe S.D., Kulkarni V.S., Non-homogeneous steady state heat conduction problem in a thin circular plate and thermal stresses, Int. J. Thermophysics, 30, 1688-1696, (2009.)

[5] Ozisik M.N., Boundary value problems of heat conduction, International Textbook Company, Scranton, Pennsylvania, 148-163, (1968).

[6] Nowinski J.L., Theory of thermoelasticity with applications, Sijthoff International Publishers B.V. Alphen aan den Rijn, The Netherlands, 407, (1978) 